\title{
Metabolomic profiling in the prediction of gestational diabetes mellitus
}

\author{
Rhonda Bentley-Lewis • Jennifer Huynh • Grace Xiong • \\ Hang Lee • Julia Wenger • Clary Clish • David Nathan • \\ Ravi Thadhani $\cdot$ Robert Gerszten
}

Received: 31 December 2014 / Accepted: 20 February 2015 / Published online: 7 March 2015

(C) Springer-Verlag Berlin Heidelberg 2015

\begin{abstract}
Aims/hypothesis Metabolomic profiling in populations with impaired glucose tolerance has revealed that branched chain and aromatic amino acids (BCAAs) are predictive of type 2 diabetes. Because gestational diabetes mellitus (GDM) shares pathophysiological similarities with type 2 diabetes, the metabolite profile predictive of type 2 diabetes could potentially identify women who will develop GDM.

Methods We conducted a nested case-control study of 18- to 40-year-old women who participated in the Massachusetts General Hospital Obstetrical Maternal Study between 1998 and 2007. Participants were enrolled during their first trimester of a singleton pregnancy and fasting serum samples were collected. The women were followed throughout pregnancy and identified as having GDM or normal glucose tolerance (NGT) in the third trimester. Women with GDM $(n=96)$ were
\end{abstract}

Electronic supplementary material The online version of this article (doi:10.1007/s00125-015-3553-4) contains peer-reviewed but unedited supplementary material, which is available to authorised users.

R. Bentley-Lewis $(\bowtie) \cdot J$. Huynh $\cdot$ G. Xiong $\cdot$ D. Nathan Department of Medicine/Diabetes Unit, Massachusetts General Hospital, 55 Fruit Street, Bulfinch 4-415, Boston, MA 02114, USA

e-mail: rbentleylewis@mgh.harvard.edu

H. Lee

Biostatistics Center, Massachusetts General Hospital, Boston, MA, USA

J. Wenger $\cdot$ R. Thadhani

Department of Medicine/Nephrology, Massachusetts General

Hospital, Boston, MA, USA

C. Clish

Broad Institute of MIT and Harvard, Cambridge, MA, USA

R. Gerszten

Department of Medicine/Cardiology, Massachusetts General

Hospital, Boston, MA, USA matched to women with NGT ( $n=96$ ) by age, BMI, gravidity and parity. Liquid chromatography-mass spectrometry was used to measure the levels of 91 metabolites.

Results Data analyses revealed the following characteristics (mean $\pm \mathrm{SD}$ ): age $32.8 \pm 4.4$ years, BMI $28.3 \pm 5.6 \mathrm{~kg} / \mathrm{m}^{2}$, gravidity $2 \pm 1$ and parity $1 \pm 1$. Six metabolites (anthranilic acid, alanine, glutamate, creatinine, allantoin and serine) were identified as having significantly different levels between the two groups in conditional logistic regression analyses $(p<0.05)$. The levels of the BCAAs did not differ significantly between GDM and NGT.

Conclusions/interpretation Metabolic markers identified as being predictive of type 2 diabetes may not have the same predictive power for GDM. However, further study in a racially/ethnically diverse population-based cohort is necessary.

Keywords Amino acids · Gestational diabetes mellitus · Metabolite profiling $\cdot$ Metabolites $\cdot$ Metabolomics

$\begin{array}{ll}\text { Abbreviations } \\ \text { BCAA } & \text { Branched chain and aromatic amino acids } \\ \text { GDM } & \text { Gestational diabetes mellitus } \\ \text { GLT } & \text { Glucose load test } \\ \text { MGH } & \text { Massachusetts General Hospital } \\ \text { NGT } & \text { Normal glucose tolerance }\end{array}$

Introduction

Gestational diabetes mellitus (GDM), defined as diabetes diagnosed during pregnancy that is not clearly overt diabetes, occurs in approximately 5-6\% of pregnancies in the USA [1]. Early identification of women at risk for GDM is critical in order to take advantage of GDM risk-reduction strategies and 
to minimise the detrimental consequences of GDM for mother and offspring. Therefore, research efforts have targeted firsttrimester data collection. In addition to clinical predictors, such as race/ethnicity, family history, BMI and prior history of GDM, first-trimester biomarkers, such as lower levels of adiponectin and sex-hormone-binding globulin, strengthened a GDM risk prediction model [2]. In addition, high levels of C-reactive protein in the first trimester have been associated with an increased risk of GDM [3]. However, studies examining first-trimester metabolites with the aim of identifying women at risk for GDM have been limited. Consequently, we believe our research will further demonstrate the relevance of this area of investigation to GDM risk prediction.

Metabolomic profiling, the systematic study of smallmolecule products of biochemical pathways, has been used to predict, diagnose and monitor several metabolic disorders, including GDM [4]. Yet, the findings of previous metabolomic studies that attempted to determine biomarkers for diagnosing GDM at 14-25 weeks' gestational age have been inconsistent [4]. When considering other potentially relevant models, a recent metabolomic study in a population with impaired glucose tolerance identified the branched chain and aromatic amino acids (BCAAs) leucine, isoleucine, valine, tyrosine and phenylalanine as being predictive of incident type 2 diabetes [5]. Because GDM is associated with an increased risk of subsequent type 2 diabetes [1] we hypothesised that the metabolite profile predictive of type 2 diabetes would also identify women in their first trimester of pregnancy who would develop GDM.

\section{Methods}

We conducted a nested case-control study of women who presented for prenatal care to the Massachusetts General Hospital (MGH) Obstetrical Department between September 1998 and January 2007 and participated in the MGH Obstetrical Maternal Study, a previously described population [3]. Women were included in our study if they met the following criteria: were aged 18-40 years; self-identified as white race; delivered live infants at full term (gestational age $\geq 37$ weeks); were healthy, including no history of pregestational diabetes, hypertension or cardiac disease prior to delivery and had complete OGTT data in the third trimester. Approval from the institutional review board was granted by the Partners Human Research Committee prior to study initiation and all study participants provided informed written consent at enrolment.

Participants provided fasting serum samples, and demographic and clinical information, at the first prenatal visit. Women were identified as having GDM or normal glucose tolerance (NGT) based on their third-trimester glucose load test (GLT) and OGTT results. Women were diagnosed with
GDM by a $1 \mathrm{~h} 50 \mathrm{~g}$ GLT value $\geq 7.8 \mathrm{mmol} / \mathrm{l}$ and two abnormal values for a $3 \mathrm{~h} 100 \mathrm{~g}$ OGTT according to Carpenter-Coustan criteria [1]. NGT was defined by a screening $1 \mathrm{~h} 50 \mathrm{~g}$ GLT value $<7.8 \mathrm{mmol} / 1$. The Broad Institute of the Massachusetts Institute of Technology and Harvard performed all targeted metabolomic analyses using liquid chromatography-mass spectrometry based on methodology described by Wang et al [5] in conjunction with available platform metabolites.

All women identified with GDM were included in the study $(n=96)$ and were matched 1:1 to women with NGT $(n=96)$ by age, BMI, gravidity and parity. Because BMI was hypothesised to be most closely correlated with our metabolites, we first divided GDM and control populations into two BMI subgroups: $<30 \mathrm{~kg} / \mathrm{m}^{2}$ (range 19.1-29.9 kg/m ${ }^{2}$ ) and $\geq 30 \mathrm{~kg} / \mathrm{m}^{2}$ (range $30.0-57.7 \mathrm{~kg} / \mathrm{m}^{2}$ ). Next, gravidity was matched within a variance of $0-4$ and then parity was matched within a variance of $0-2$. Finally, age was matched within the range of $18.4-43.1$ years in the BMI $<30 \mathrm{~kg} / \mathrm{m}^{2}$ group and $23.4-44.1$ years in the BMI $\geq 30 \mathrm{~kg} / \mathrm{m}^{2}$ group.

Study population characteristics were described by number (percentage) for categorical variables and mean $\pm \mathrm{SD}$ for continuous variables. Paired samples $t$ tests were conducted to compare the difference in means of women with GDM and NGT for each metabolite. A theoretical study sample size of 110 women with GDM and 110 women with NGT was determined to provide a $>90 \%$ power at a multiple comparison adjusted $\alpha(p=0.005)$ to detect a statistical difference in means between the two groups. The effect size, measured as Cohen's $d>0.583$, was even smaller than the acceptable magnitude of clinically meaningful differences $(d>0.75)$. Because we were unable to detect differences in metabolites when applying stringent multiple comparisons corrections, we used a twotiered approach. That is, for those metabolites that achieved statistical significance at the unadjusted $\alpha(p<0.05)$, we then conducted conditional logistic regression analyses to estimate the association between each metabolite and the odds of developing GDM. All statistical analyses were performed using SAS for Windows, version 9.2, statistical software (SAS Institute, Cary, NC, USA).

\section{Results}

Baseline characteristics revealed that systolic and diastolic blood pressure and birthweight were similar between the two groups (Table 1). Women with GDM gained significantly less weight and had their first prenatal visit and delivery at earlier gestational ages than women with NGT.

Ninety-one metabolites, including amino acids, amines and other polar metabolites, were analysed (see electronic supplementary material [ESM] Table 1). In univariate paired 
Table 1 Baseline characteristics for women with GDM vs women without GDM
Values are presented as $n(\%)$ or mean \pm SD

\begin{tabular}{|c|c|c|c|}
\hline Characteristic & $\operatorname{GDM}(n=96)$ & $\begin{array}{l}\text { No GDM } \\
(n=96)\end{array}$ & $p$ value \\
\hline Age (years) & $33.3 \pm 5.0$ & $32.4 \pm 3.7$ & 0.14 \\
\hline BMI $\left(\mathrm{kg} / \mathrm{m}^{2}\right)$ & $28.2 \pm 6.3$ & $28.5 \pm 4.7$ & 0.70 \\
\hline Systolic blood pressure (mmHg) & $116 \pm 11$ & $115 \pm 9$ & 0.29 \\
\hline Diastolic blood pressure $(\mathrm{mmHg})$ & $73 \pm 8$ & $71 \pm 7$ & 0.09 \\
\hline Live births & $0.6 \pm 0.8$ & $0.7 \pm 0.8$ & 0.79 \\
\hline Total pregnancies & $2.4 \pm 1.5$ & $2.3 \pm 1.4$ & 0.76 \\
\hline Gestational age at first prenatal visit (weeks) & $10.4 \pm 2.4$ & $11.7 \pm 3.4$ & 0.003 \\
\hline Gestational age at delivery (weeks) & $38.8 \pm 1.8$ & $39.8 \pm 1.0$ & $<0.001$ \\
\hline Gestational weight gain (kg) & $10.4 \pm 4.9$ & $13.6 \pm 5.2$ & $<0.001$ \\
\hline Baby weight (g) & $3,486.9 \pm 577.7$ & $3,593.0 \pm 441.1$ & 0.16 \\
\hline Birthweight for gestational age percentile (\%) & $60.3 \pm 27.0$ & $58.9 \pm 27.2$ & 0.72 \\
\hline Pre-eclampsia $(\%)$ & $2.1(2)$ & $0.0(0)$ & 0.16 \\
\hline Total cholesterol (mmol/l) & $5.4 \pm 1.2$ & $5.2 \pm 0.6$ & 0.52 \\
\hline HDL-cholesterol (mmol/l) & $1.6 \pm 0.3$ & $1.7 \pm 0.4$ & 0.60 \\
\hline LDL-cholesterol (mmol/1) & $2.7 \pm 0.7$ & $2.8 \pm 0.5$ & 0.51 \\
\hline Triacylglycerol (mmol/l) & $2.2 \pm 1.5$ & $1.3 \pm 0.7$ & 0.02 \\
\hline Smoking status (\%) & & & 0.38 \\
\hline Never smoked & $27.1(26)$ & $36.5(35)$ & \\
\hline Past smoker & $28.1(27)$ & $22.9(22)$ & \\
\hline Current smoker & $4.2(4)$ & $6.3(6)$ & \\
\hline Breast feeding at discharge (\%) & $70.8(68)$ & $67.7(65)$ & 0.60 \\
\hline Storage time of serum samples (days) & $3,819 \pm 625$ & $3,725 \pm 713$ & 0.34 \\
\hline
\end{tabular}

analyses, levels of anthranilic acid, alanine, glutamate, allantoin and serine were found to be higher and creatinine was lower in women with GDM than in women with NGT (Table 2). The BCAAs did not differ significantly between the GDM and NGT populations. However, because gestational age at first prenatal visit was statistically different between GDM and NGT populations, we adjusted for gestational age at first prenatal visit. After adjustment, the odds ratios achieved statistical significance and notably increased for anthranilic acid $(p<0.01)$ and glutamate $(p<0.01)$ (Table 2).

\section{Discussion}

Of the 91 metabolites that were analysed, levels of anthranilic acid, alanine, glutamate, allantoin and serine were found to be significantly higher and creatinine significantly lower in women who subsequently developed GDM when compared with women with NGT. Because the odds ratios were appreciable, further investigation is warranted in larger, more racially/ ethnically diverse populations to determine the utility of these metabolites in predicting GDM. In fact, the metabolites we observed to be significantly associated with GDM in

Table 2 Metabolite comparisons by GDM status

\begin{tabular}{|c|c|c|c|c|c|c|c|}
\hline \multirow[t]{2}{*}{ Metabolite } & \multicolumn{2}{|l|}{ Metabolite level $^{\mathrm{a}}$} & \multirow[t]{2}{*}{$p$ value } & \multirow[t]{2}{*}{ OR $(95 \% \mathrm{CI})$} & \multirow[t]{2}{*}{$p$ value } & \multirow{2}{*}{$\begin{array}{l}\text { OR }(95 \% \mathrm{CI}) \text { adjusted for } \\
\text { gestational age at first } \\
\text { prenatal visit }\end{array}$} & \multirow[t]{2}{*}{$p$ value } \\
\hline & $\operatorname{GDM}(n=96)$ & No GDM $(n=96)$ & & & & & \\
\hline Anthranilic acid & $3,010.91 \pm 2,553.26$ & $2,600.42 \pm 2,551.96$ & 0.01 & $1.61(1.12,2.32)$ & 0.01 & $1.78(1.19,2.65)$ & $<0.01$ \\
\hline Alanine & $1,304,430.64 \pm 1,265,746.06$ & $1,218,963.32 \pm 1,199,902.28$ & 0.01 & $1.54(1.11,2.14)$ & 0.01 & $1.53(1.13,2.11)$ & 0.01 \\
\hline Glutamate & $388,114.19 \pm 372,673.04$ & $344,942.44 \pm 320,402.69$ & 0.03 & $1.40(1.03,1.90)$ & 0.03 & $1.50(1.08,2.08)$ & $<0.01$ \\
\hline Creatinine & $1,137,860.68 \pm 1,141,200.95$ & $1,197,551.86 \pm 1,189,022.39$ & 0.03 & $0.68(0.49,0.95)$ & 0.02 & $0.80(0.69,0.92)$ & 0.01 \\
\hline Allantoin & $115,523.86 \pm 77,751.24$ & $85,937.75 \pm 62,074.51$ & 0.03 & $1.38(1.00,1.88)$ & 0.05 & $1.42(1.01,2.00)$ & 0.05 \\
\hline Serine & $444,402.40 \pm 440,922.76$ & $417,080.91 \pm 410,455.66$ & 0.03 & $1.50(1.07,2.12)$ & 0.02 & $1.65(1.13,2.41)$ & 0.01 \\
\hline
\end{tabular}

${ }^{a}$ Metabolite levels were standardised to SD units 
unadjusted analyses do have relevance to glucose-mediated pathways. For example, glutamate [6] and creatinine [7] have been associated with the development of hyperglycaemia and alanine is an important substrate for gluconeogenesis [8].

The strengths of this study highlight the methodological rigour. We used biochemical confirmation to define our GDM and NGT groups. Also, because fasting serum samples were collected in the first trimester and women with GDM were well matched to those with NGT, potential confounding by existing glucose intolerance was theoretically minimised. However, first-trimester glucose values were unavailable for examination. Although gestational weight gain was significantly different between the two groups, we did not adjust the odds ratios for this variable since we aimed to examine first-trimester predictors of GDM. Additionally, limited lipid data prevented adjustment for triacylglycerols to examine their potential impact on our findings.

Although several limitations to our study exist, we believe our methodological approach enhanced our study findings. Our study could have been limited by correlations between metabolites and clinical variables. For example, we observed that glutamate was correlated with BMI, gravidity and parity and that serine was correlated with BMI and parity (data not shown). Because metabolites have been correlated with age [9] and BMI [10], the associations we observed between metabolites and the odds of developing GDM may have been underestimated because we matched on these variables. However, the potential underestimation of our findings enhances our confidence in our results. Additionally, we recognise that the inclusion of only women identifying as 'white' limited the generalisability of our results. However, to optimally focus our study on differences in GDM status, we chose to examine women of one race. Additionally, the high degree of collinearity among the metabolites prevented the performance of cluster analyses and potential identification of specific metabolic pathways. Moreover, although there was no significant difference between GDM and NGT groups in the length of time that the samples were stored before analysis, validation studies to determine the effect of storage time on metabolite levels were not performed. Consequently, further investigation in a racially/ ethnically diverse population-based cohort is necessary to determine the usefulness of these metabolites in predicting GDM. Future prospective studies should also involve an untargeted metabolomic assay to capture as many metabolite differences as possible, including lipids, as well as include family history of diabetes and glucose levels at the first prenatal visit.

To our knowledge, this is the first study to examine the potential of first-trimester metabolites in identifying women at risk for GDM. Given the growing incidence of obesity and diabetes in the USA, early detection of GDM provides an opportunity for primary intervention strategies which would not only improve the health of mother and fetus but also decrease the risk of development of type 2 diabetes. These metabolite data facilitate hypothesis generation regarding the pathogenesis of GDM and have the potential to guide future research into novel metabolomic biomarkers of GDM.

Funding This study was supported in part by the following grants: National Institutes of Health R03 DK096152 awarded to RB-L and K24 DK094872 awarded to RT and the Robert Wood Johnson Foundation Harold Amos Medical Faculty Development Program and Massachusetts General Hospital Executive Committee on Research/ Multicultural Affairs Office Physician Scientist Development Award awarded to RB-L.

Duality of interest The authors declare that there is no duality of interest associated with this manuscript.

Contribution statement RB-L participated in hypothesis generation, study design, statistical analysis planning, data interpretation and manuscript development (draft and revisions). JH participated in statistical analysis planning, data interpretation and manuscript development (draft and revisions). GX participated in acquisition of data, data management and manuscript review. HL participated in statistical analysis planning and execution, data interpretation and manuscript review. JW participated in statistical analysis planning and manuscript review. CC, RT RG and DN participated in hypothesis generation, study design, data acquisition and manuscript review. All authors had final approval of the version to be published. RB-L is the guarantor of this work, had full access to all of the data in the study and takes responsibility for the integrity of the data and the accuracy of the data analysis.

\section{References}

1. American Diabetes Association (2014) Standards of medical care in diabetes-2014. Diabetes Care 37(suppl 1):S14-S80

2. Nanda S, Savvidou M, Syngelaki A, Akolekar R, Nicolaides KH (2011) Prediction of gestational diabetes mellitus by maternal factors and biomarkers at 11 to 13 weeks. Prenat Diagn 31:135-141

3. Wolf M, Sandler L, Hsu K, Vossen-Smirnakis K, Ecker JL, Thadhani R (2003) First-trimester C-reactive protein and subsequent gestational diabetes. Diabetes Care 26:819-824

4. Huynh J, Xiong G, Bentley-Lewis R (2014) A systematic review of metabolite profiling in gestational diabetes mellitus. Diabetologia 57: 2453-2464

5. Wang TJ, Larson MG, Vasan RS et al (2011) Metabolite profiles and the risk of developing diabetes. Nat Med 17:448-453

6. Di Cairano ES, Davalli AM, Perego L et al (2011) The glial glutamate transporter 1 (GLT1) is expressed by pancreatic beta-cells and prevents glutamate-induced beta-cell death. J Biol Chem 286:1400714018

7. Harita N, Hayashi T, Sato KK et al (2009) Lower serum creatinine is a new risk factor of type 2 diabetes: the Kansai healthcare study. Diabetes Care 32:424-426

8. Waterhouse C, Keilson J (1978) The contribution of glucose to alanine metabolism in man. J Lab Clin Med 92:803-812

9. Soda E, Miura I, Hoshino H et al (2014) Impacts of age on plasma monoamine metabolite concentrations in a large cohort of healthy individuals. Psychiatry Res 220:639-645

10. Gogna N, Krishna M, Oommen AM, Dorai K (2015) Investigating correlations in the altered metabolic profiles of obese and diabetic subjects in a South Indian Asian population using an NMR-based metabolomic approach. Mol BioSyst 11:595-606 